\title{
FACTORES CONDICIONANTES QUE PREDISPONEN A LA INFECCIÓN URINARIA EN GESTANTES EN EL PUESTO DE SALUD "CONO NORTE" CIUDAD NUEVA - TACNA - 2007
}

Responsable: Med. G.O. José Ferrer Rojas

\section{RESUMEN}

El presente estudio permitió conocer las características, riesgo y comportamiento de la infección urinaria asociada al embarazo en el Puesto de Salud "Cono Norte", Ciudad Nueva Tacna, Perú. El diseño de investigación es retrospectivo, transversal descriptivo. Para tal efecto se revisaron 69 historias clínicas de embarazadas con I.T.U. de un total de 224 partos en el año 2007. En conclusión, la infección urinaria fue más frecuente entre los 15 y 25 años (48) y hasta las 20 semanas de embarazo (43); 47 casos con signos patológicos (flujo vaginal), la mayoría de la embarazadas presentaron un parto eutocico (18), cesáreas (14), gestación en curso (37). El antibiótico de primera elección amoxicilina, en segundo lugar la gentamicina.

\section{ABSTRACT}

The present study allowed to know the characteristics, risk and behavior of urinary infection associated to the pregnancy in Healthcare Post 'Cono Norte,' Ciudad Nueva - Tacna, Peru. The research design is retrospective, descriptive cross. To this effect, it was necessary review the medical records of 69 pregnant women with urinary infection from a total of 224 births in 2007. In conclusion, the urinary infection was more frecuent between 15 and 25 years (48) and until 20 weeks of pregnancy (43); 47 cases with pathological signs (vaginal flow), most of the pregnant women showed a eutocic childbirth (18), cesarean (14), ongoing pregnancy (37). The antibiotic of first choice was amoxicillin , in second choice was gentamicin.

\section{INTRODUCCION}

La infección de las vías urinarias constituye una de las más frecuentes durante el embarazo. Los microorganismos involucrados son principalmente las enterobacterias, con un $(80 \%)$ la Escherichia-coli, Klebsiella, Proteus mirabilis, enterobacter. Existen además otros agentes que siguen en frecuencia como el Streptococo y el Stafilococcus coagulasa negativo.

Durante el embarazo se producen modificaciones anatómicas y funcionales que aumentan el riesgo a padecer una infección urinaria. Entre ellas se destacan: la hidronefrosis del embarazo, el aumento del volumen urinario en los uréteres que produce una columna líquida continua que ayuda a la propagación de la infección desde la vejiga al riñón, disminución del tono ureteral y vesical que se asocia a un aumento del volumen urinario en la vejiga, aumentando su capacidad vesical y disminuyendo su vaciamiento (estasis urinaria), obstrucción parcial del uréter por el útero gravido y rotado hacia la derecha, aumento de P.H: de la orina, especialmente por excreción aumentada de bicarbonato que favorece la multiplicación bacteriana, hipertrofia de la musculatura longitudinal del uréter, aumento de la filtración glomerular que determina la presencia de glucosa en la orina, lo que favorece la aparición de los gérmenes, aumento del reflujo vesicoureteral, menor capacidad de defensa del epitelio del aparato urinario bajo, incremento de la secreción urinaria de estrógenos y el ambiente hipertónico de la médula renal.
Si no existen enfermedades concomitentes, el riesgo es mayor en las embarazadas de mayor edad, multiparas y de bajo nivel socioeconómico, pero sobre todo en aquellas con historia previa de infección urinaria. Del 2 al $10 \%$ de las embarazadas sin antecedentes desarrollan bacteriuria asintomática y sin tratamiento; el 30 al $50 \%$ evolucionarán a pielonefritis, ésta por su parte puede asociarse a insuficiencia renal aguda, sepsis y shock séptico. Aumenta el riesgo prematuro y R.N. de bajo peso al nacer. La mortalidad fetal más alta se presenta cuando la infección ocurre durante los 15 días que anteceden al parto. Por lo expuesto anteriormente, la detección y el tratamiento temprano de la I.T.U. en las embarazadas debe ser una prioridad.

\section{MATERIALYMÉTODOS}

1. Lugar de Ejecución:El estudio se realizó durante el año 2007 en el Puesto de Salud "Cono Norte".

2. Muestra de Estudio:La muestra de estudio fue de 69 historias clínicas de embarazadas con infección urinaria, durante el periodo del 2007 en el Puesto de Salud de Cono Norte.

3. Recolección de datos:Se recopiló la información de una fuente secundaria, se realizó un análisis de selección y vaciado de los datos de las historias clínicas que corresponden a gestantes con infección urinaria.

4. Procesamiento y análisis de los datos:Se utilizó SPSS versión 10 en español, 
procesador de textos Microsoft Word, Programa Excel.

\section{RESULTADOS}

Cuadro 01: Puesto de Salud "Cono Norte" Ciudad Nueva Tacna, prevalencia de infección urinaria en el embarazo año 2007.

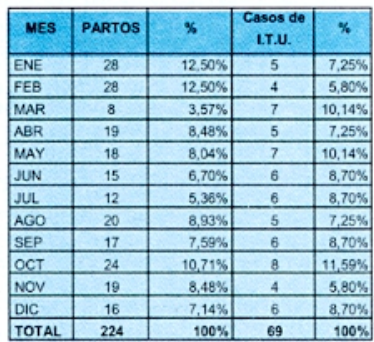

Cuadro 02: Puesto de Salud "Cono Norte" Ciudad Nueva Tacna, según la edad materna en el año 2007.

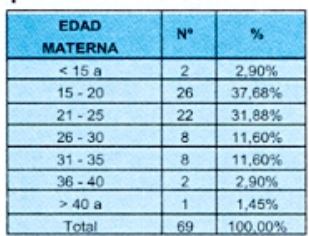

Cuadro 03: Puesto de Salud "Cono Norte" Ciudad Nueva Tacna, según la edad gestacional.

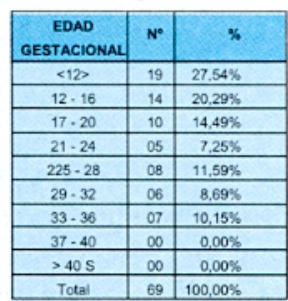

Cuadro 04: Puesto de Salud "Cono Norte" Ciudad Nueva Tacna, según los signos y síntomas para el año 2007.

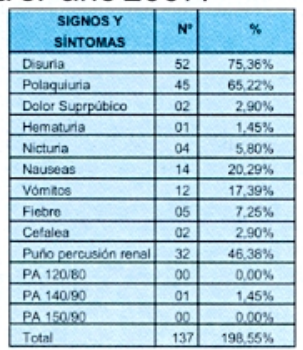

Cuadro 05: Puesto de Salud "Cono Norte" Ciudad Nueva Tacna, según el término del embarazo para el año 2007.

\begin{tabular}{|c|c|c|}
\hline $\begin{array}{c}\text { TERMINO DEL } \\
\text { EMBARAZO }\end{array}$ & No & $\%$ \\
\hline Parto espontáneo & 18 & $26,09 \%$ \\
\hline Cesáreas & 14 & $20,29 \%$ \\
\hline Gestación en curso & 37 & $53,62 \%$ \\
\hline Total & 69 & $100,00 \%$ \\
\hline
\end{tabular}

Cuadro 06: Puesto de Salud "Cono Norte" Ciudad Nueva Tacna, según el uso de antibióticos para el año 2007.

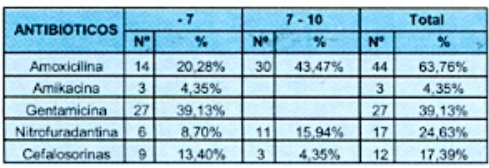

\section{IV.DISCUSION}

Según el estudio realizado, al distribuir las pacientes portadoras de I.T.U. en embarazo no se corresponde con las literaturas encontradas, pues, se demostró que la edad promedio de aparición fue de 15 a 22 años. Se estipula la existencia de partos pretérminos más frecuente en niños nacidos con I.T.U., lo cual todavía nosotros no tenemos conocimiento al respecto, asimismo coincidimos con la literatura respecto al tipo de parto, pues la gran mayoria fueron eutocicos. Valoramos en esta revisión que la incidencia del bajo peso al nacer es mínima en relación con otras publicaciones.

Reconocemos que la morbilidad perinatal es válida al igual que el distress respiratorio, son entidades que afectan al bajo peso. La morbilidad asociada (crónicas) al embarazo está relacionada con la morbilidad materna de acuerdo con el daño que puede producir ell.T.U, $u$ otras enfermedades asociadas.

\section{CONCLUSIONES}

La infección urinaria es más frecuente entre los 15 y 22 años. 20 semanas de embarazo (43 casos con signos patológicos (flujo vaginal). La mayoría de las embarazadas presentaron un parto eutocico. El antibiótico de primera elección amoxicilina, en segundo lugar la gentamicina.

\section{VI.RECOMENDACIONES}

Después de la exposición de esta investigación realizada sobre infección urinaria en el embarazo, vemos con mucha preocupación la falta de equipos indispensables para mejorar el funcionamiento de este Puesto de Salud en el "Cono Norte" Ciudad Nueva y la necesidad de un laboratorio con una implementación para hacer los exámenes correspondientes y beneficiar a los pobladores de esta zona.

Además, contar con personal entrenado para ayudar a practicar un trabajo responsable en beneficio de estos pacientes, todo cual es responsabilidad de las autoridades de salud.

\section{BIBLIOGRAFÎA}

Pérez, A . y Donoso Siña, A.(1999). Obstetricia. 2da. edición, pág. 424.

Cifuentes B., Rodrigo. (1988), Obstetrícia de alto riesgo. 2 da. edición, pág. 271.

Benson, Ralph.( 1989). Gineco obstétrico. Tomo I, Edit. Manual Moderno.

Schwarz,Ricardo.(1991), Obstetricia. edit. Ateneo y Pacheco Jose.(1999), Segunda edición.

Ticona Rendón, Manuel(1999). Medicina Perinatal Prevención Diagnóstico y Tratamiento. 


\section{MIEMBROS DEL CONSEJO DE COORDINACIÓN DE INVESTIGACIÓN DE LA UNIVERSIDAD NACIONAL JORGE BASADRE GROHMANN 2007}

FACULTADDE INGENIERIADEMINAS

FACULTAD DE INGENIERÍA METALÚRGICA

FACULTAD DEINGENIERÍAPESQUERA

FACULTADDE CIENCIAS AGRICOLAS

FACULTAD DE INGENIERÍA EN INDUSTRIASALIMENTARIAS

FACULTADDE OBSTETRICIA

FACULTADDE CIENCIAS DELAEDUCACIÓN

FACULTADDE CIENCIAS

FACULTAD DEENFERMERÍA

FACULTAD DEARTEURBANISMOYARQUITECTURA

FACULTAD DE CIENCIAS MÉDICAS

FACULTAD DELETRASYCIENCIAS JURÍDICAS

FACULTAD DE INGENIERIAA

FACULTAD DE CIENCIAS CONTABLESYFINANCIERAS

FACULTAD DE CIENCIASADMINISTRATIVAS
Ing. Julio Vargas Paniagua

Ing. Freddy Felipe Cori Nina

Blgo. Pesq. Nelver Coronel Flores

Dr. Oscar Octavio Fernádez Cutire

Mgr. Nicolas Guillermo Sequeiros Flores

Obst. Juana Inés Barrera Grados

Lic. Santos Isidro Conde Lucero

Mgr. Dionicio Miltón Chávez Muñoz

Mgr. Isabel Rodríguez Monzón

Arq. Jorge Luis Espinoza Molina

Dr. Manuel Ticona Rendón

Mgr. Maria Ruth Rozas Flores

Ing. Omar Moisés Dueñas Rospigliosi

Mgr. Rolando Wilman Vázquez Jaico

Cristóbal Santiago Laura Vargas

Rosa Isabel Espinoza Francia

Yovana Verónica Mamani Calderón 